\title{
Current Measurements in High Performance Polymers Used in Aeronautic Cables
}

\author{
El Hadji Ndongo Diaw, Séverine Le Roy and Gilbert Teyssèdre \\ LAPLACE, Université Paul Sabatier, CNRS, INPT, UPS
}

118 route de Narbonne - bât 3R3 - 31062 Toulouse cedex 9 France

\section{Eddy Aubert}

Technical and Innovation Department Safran Electrical Power, Parc d'activité d'Andromède 1 rue Louis Blériot, CS 80049, 31702 Blagnac cedex - France

\begin{abstract}
This article presents experimental characterisation of PI (polyimide, Kapton-FN) and PTFE (polytetrafluoroethylene), two polymers used as insulators in aeronautic cables. Current measurements are performed for different electric fields and temperatures, and the current behaviour is analysed with the help of a conductivity law. We then discuss the conductivity law and its optimized parameters.
\end{abstract}

Index Terms - HVDC aeronautic cables, polymers, PTFE, Polyimide, current measurement

\section{INTRODUCTION}

THE actual main challenge in aeronautic applications is to propose a more electrical aircraft. This (r)evolution is driven by two aspects:

An economic one: the use of electrical energy instead of pneumatic or hydraulic energy decreases the weight of an aircraft, which in turn will decrease the fuel consumption ${ }^{1}$. Moreover, electrical energy requires less maintenance compared to hydraulic and pneumatic energies.

An ecological aspect: there is a trend to use new and hybrid energies such as hydrogen fuel cells, solar... in order to reduce the ecological footprint.

Three types of energy sources are actually in use in an aircraft to operate auxiliary functions [1]: electric (for navigation instruments, actuator controls, etc), pneumatic (for anti-icing and de-icing system as an example), and hydraulic (for actuation system, flight controls, landing gears and thrust reversers)

The perspective of a more electrical aircraft implies a necessary increase of the embedded power to replace hydroelectric and pneumatic energy by electric energy. DC network seems a potential candidate and has advantages over $\mathrm{AC}$ : the transmitted power is higher for the same cable dimension, and presents fewer losses. Low voltage DC $(<28 \mathrm{~V})$ has been used for long in the civil aeronautics. This last decade has seen the development of aircrafts (A350, B787) with more embedded electric energy for passenger's comfort but also in avionics (bleedless air regulation, engine start or even brakes controllers [2]).

This increase in power level pushes the actual systems to their limits. This is particularly the case for DC cables transmitting the electricity through the aircraft, with environmental constraints that are pressure, temperature, and electrical stress, depending on the area where the cable is located. There are no specific HVDC cables in aeronautics, as it is the case for terrestrial HVDC. The actual cables used for AC and for $270 \mathrm{~V}$ DC are the ones that are thought to be used for HVDC (i.e. $>540$ VDC). In the future, the prospects will be oriented to further increase this voltage level up to $3 \mathrm{kV}$ DC based on existing technologies. Hence, there is a need to understand the dielectric behaviour of the insulating materials constitutive of the aeronautic cables.

DC network presents an advantage over AC in that partial discharge phenomena, which are a real concern for increasing the service voltage in aerospace field [3], are in principle much less active. But, contrary to $\mathrm{AC}$ regime, dominated by the capacitive field, under DC, the electric field distribution is driven by the electrical conductivity of the materials. The latter varies in a much more substantial way as a function of temperature, electric field, or material than permittivity. As a result, the field distribution is much more difficult to anticipate.

Looking at the literature on HVDC cables for the submarine or terrestrial transmission of electricity, where polyethylene $(\mathrm{PE})$ is the main insulating material, the electrical conductivity is strongly dependent upon electric field (non-linear behaviour) and temperature. Moreover, many works [4] have revealed the 
accumulation of space charge in the insulator, leading to local strengthening of the electric field compared to the geometric field. The same kind of conclusion is encountered in the literature for materials such as polyimide (PI) or polytetrafluoroethylene (PTFE) [5-7], that are materials also used as insulation in domains such as microelectronics or space applications.

The aim of the present work is to characterize the behaviour of two materials used as insulators in the actual aeronautic cables, and analyse it using well known equations of the literature [8]. To do so, different experimental protocols have been used to get the widest range of electric fields and temperatures. A conductivity law is then proposed and is discussed as regards known injection/conduction processes.

\section{METHODS}

\subsection{INVESTIGATED MATERIALS}

Most of aeronautic cables are made of a combination of PI and of fluorinated polymers such as PTFE, providing the insulation respectively with high thermal and electrical insulation properties $[9,10]$ and resistance to arc tracking and fire propagation. Figure 1 shows an example of cross-section of a cable used for power transmission in aircraft. It consists of metal strands and different layers of insulation (inner layer: PI of $60 \mu \mathrm{m}$ and outer layer: PTFE of $200 \mu \mathrm{m}$ thickness) wrapped around the conductor, of rough diameter about $3.70 \mathrm{~mm}$. The exact insulation thickness depends on the number of tapes, which is not the same all over the circumference.

PI samples used in the present study were provided in the form of films of thickness ranging from 30 up to $36 \mu \mathrm{m}$. The size of each sample is approximately $4 \times 4 \mathrm{~cm}^{2}$. The type of PI used is a Kapton ${ }^{\circledR}$ FN, which is a Kapton ${ }^{\circledR}$ HN type covered on both sides by FEP (fluorinated ethylene propylene) polymer layers [11], having a thickness of $\approx 2.5 \mu \mathrm{m}$ each. This FEP layer allows having a good adhesion between PI and PTFE during cable manufacturing.

PTFE samples used were also provided in the form of films of thickness ranging from 72 up to $77 \mu \mathrm{m}$. The size of each sample is approximately $4 \times 4 \mathrm{~cm}^{2}$. As-received PTFE samples are not sintered, unlike PTFE on real aeronautic cables, which has been thermally treated during cable manufacturing. Sintering was performed in the laboratory for all PTFE samples in an oven at $360^{\circ} \mathrm{C}$ for 5 to $10 \mathrm{~min}$ in order to obtain samples representative of materials in operation.

\subsection{DSC MEASUREMENTS}

Differential Scanning Calorimetry (DSC) was performed on PTFE samples to verify that the thermal treatment (sintering) achieved at lab scale provides a material with the same structural properties as the PTFE used for aeronautic cables, with sintering realized during cable production. This is also to verify if the sintering conditions have an impact on the physical properties of PTFE. Sintering does not change the structure of PI, so DSC measurements are not proposed for PI in the present paper. Information such as the melting, crystallization and glass transition temperatures of the sample can be determined. Measurements were achieved using a TA DSC 2010 CE differential scanning calorimeter, using samples having a weight of $8 \mathrm{mg}$. For each measurement, two temperature scans

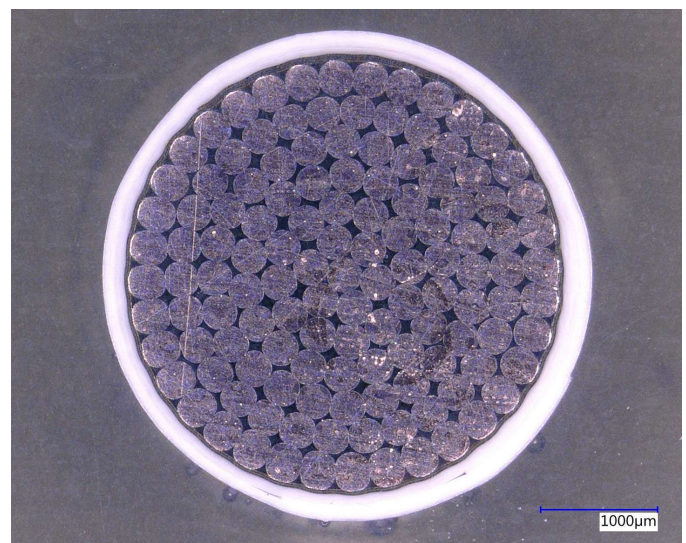

Figure 1. Example of cross-section of an aeronautic cable (DR8). The inner black layer is taped polyimide insulation, $60 \mu \mathrm{m}$ thick; the outer layer is taped PTFE, $200 \mu \mathrm{m}$ thick. The rough diameter of the conductor is $3.70 \mathrm{~mm}$.

are performed, the first to erase the "thermal history" of the sample and the second to provide information on the thermal transitions. For this study, temperature ranges from $-50{ }^{\circ} \mathrm{C}$ to $400{ }^{\circ} \mathrm{C}$ for a temperature ramp of $10^{\circ} \mathrm{C} / \mathrm{min}$ with a return to 20 ${ }^{\circ} \mathrm{C}$. The degradation temperature of PTFE is $420^{\circ} \mathrm{C}$, so upper temperature is limited to $400{ }^{\circ} \mathrm{C}$ in our study.

\subsection{CURRENT MEASUREMENTS}

Current measurements were realized on PI and PTFE samples, metallized on both sides with $50 \mathrm{~nm}$-thick gold layer of diameter $20 \mathrm{~mm}$, deposited by cold plasma sputtering. A silicon ribbon is added at the periphery of the electrodes to avoid electric field enhancement due to edge phenomena, possibly leading to breakdown. PI or PTFE samples are sandwiched between two plane parallel brass electrodes, and measurements are performed under air at atmospheric pressure. The current is recorded through a Keithley 617 ammeter with a $2 \mathrm{~s}$ dwelling time. Investigated materials have a really low conductivity and questions of noise level was addressed. A low ripple $35 \mathrm{kV}$ DC supply from Fug GbmH was used as voltage source. The lowest detectable current above noise was estimated to $0.2 \mathrm{pA}$ in the environment used.

Two different protocols have been applied on each material. The first protocol consists in applying, at a constant temperature, cycles of 3 hours of polarization and 3 hours of depolarization (voltage set to zero) for electric fields ranging from 4 up to $160 \mathrm{kV} / \mathrm{mm}$ for PTFE, and from 1 up to $120 \mathrm{kV} / \mathrm{mm}$ for PI. The difference of maximum applied electric field is due to the dielectric breakdown strength of each material. This protocol (cf. Figure $2 \mathrm{a}$ as an example for PTFE), performed on an automatized set-up, has been applied for temperatures of 20,50 , and $90{ }^{\circ} \mathrm{C}$. A new sample is used for each temperature.

The second protocol has been used to characterize each material at higher temperatures, on a non-automatized set-up. The protocol, presented on Figure $2 \mathrm{~b}$ has been applied for temperatures ranging from 100 up to $200^{\circ} \mathrm{C}$, and for a low $(20 \mathrm{kV} / \mathrm{mm})$ and a high applied electric field, being $100 \mathrm{kV} / \mathrm{mm}$ for PI and $140 \mathrm{kV} / \mathrm{mm}$ for PTFE. It is to note that for this protocol also, the depolarization cycle consists in applying a zero voltage at the electrode, i.e. the sample is not shortcircuited. A new sample is used for each electric field value. 


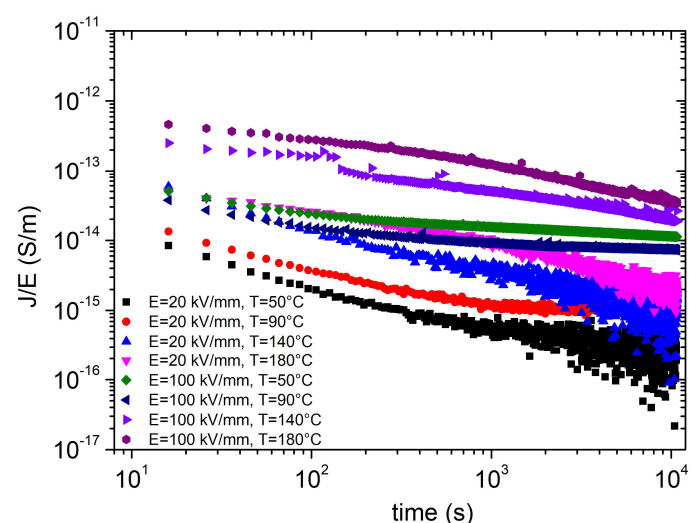

Figure 4. Normalized current densities as a function of time for different electric fields and temperatures on a $36 \mu \mathrm{m}$ thick PI film.

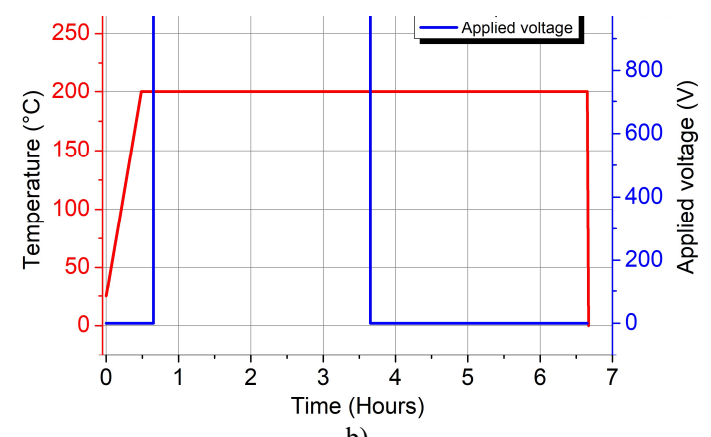

b)

Figure 2. Experimental protocols used for current measurements on PI and PTFE. a) protocol $\mathrm{n}^{\circ} 1$ consisting of cycles of polarization/depolarization of 3 hours for PTFE, for low temperatures $\left(20\right.$ up to $90^{\circ} \mathrm{C}$; b) protocol $\mathrm{n}^{\circ} 2$ consisting in applying a voltage at temperatures above $90^{\circ} \mathrm{C}$.

It is difficult in practice to obtain the steady state current in highly insulating materials. For example, Ghorbani et al. showed that the DC current in polyethylene under constant field at moderate temperature could evolve for months [12]. Stressing for long time may involve space charge build-up and field redistribution in a way that it is not clear of which field the obtained current is representative. Here, time under voltage application has been set arbitrarily to three hours, which is almost the longest time intervals published in the literature for PI $[13,15]$.

\section{EXPERIMENTAL RESULTS}

\subsection{DSC ON PTFE}

Figure 3 presents the DSC thermograms obtained from a PTFE sintered at lab scale, and a PTFE taken from a real cable, for the second temperature scan. The peaks representing the different thermal transitions for each sample appear at the same temperatures. When the sample is heated, the first characteristic temperatures are observed at $18.14^{\circ} \mathrm{C}$ and at $31.4^{\circ} \mathrm{C}$ and correspond to crystalline PTFE transitions, between triclinic and hexagonal crystalline systems [16]. The melting temperature of the sample is $324.4{ }^{\circ} \mathrm{C}$ and characterizes the disappearance of the crystalline phase of PTFE [16]. At this temperature, PTFE turns into a viscous liquid.

During cooling, a peak of crystallization is observed at a temperature of $313.7^{\circ} \mathrm{C}$, for all samples. From Figure 3, the sintered material at lab scale and the cable material have the

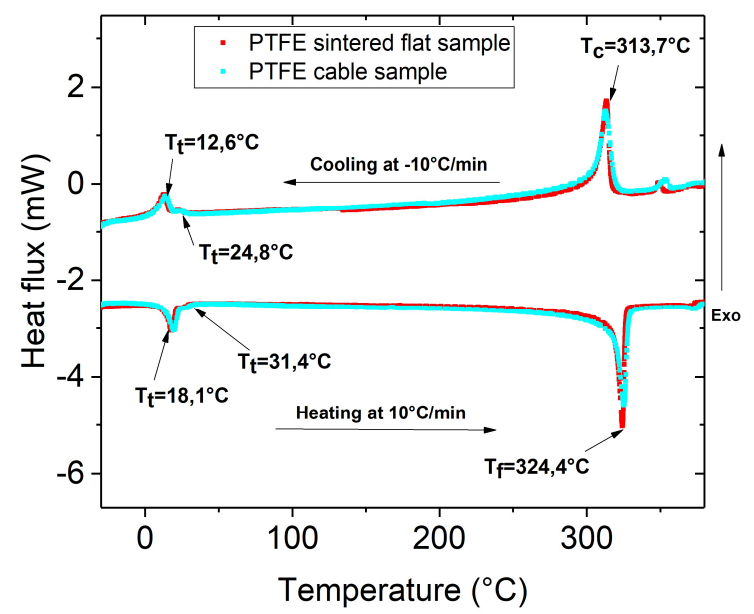

Figure 3. DSC results for PTFE samples.

same thermal transitions, and the same melting temperature. DSC also allows calculating the crystallinity of the material, by integrating the area under the curve. The crystallinity of a lab sintered material is of the order of $33 \%$ (mean of three measurements), while the one of the PTFE from cable is $33.5 \%$. The difference is within experimental error. It is to note that several sintering durations have been tested (from 5 to $8 \mathrm{~min}$ ), giving the same thermal characteristics as the one presented in Figure 3 for a lab sintered PTFE, and the same thermal transitions. The degree of crystallinity and the different thermal transitions are also consistent with data found in the literature [16]. The sintering performed in the lab allows obtaining PTFE sample having the same physical properties than the one encountered directly on an aeronautic cable.

\subsection{CURRENT MEASUREMENTS ON PI}

Figure 4 presents normalized current densities (i.e. apparent transient conductivity) as a function of time for different applied electric fields and temperatures on PI films. For each electric field (20 and $100 \mathrm{kV} / \mathrm{mm}$ ), two behaviours are observed. The first one is relevant to relatively low temperatures where the current density presents two regions: a fast decrease at short times, and a second region at longer times where the decrease of the current is slower. This behaviour is clearly observed for temperatures below $120^{\circ} \mathrm{C}$, although only 50 and $90^{\circ} \mathrm{C}$ data are presented on Figure 4. For this first behaviour, when the temperature increases (from 50 to $90^{\circ} \mathrm{C}$ ) the change in slope appears earlier in time. Comparing the data for a specific temperature, the current density increases when the electric field increases.

A second behaviour is observed for higher temperatures (140 and $180^{\circ} \mathrm{C}$ on Figure 4). For these temperatures, the current density is higher than for lower temperatures. It also presents two slopes: a first slope at short time, with a slow decrease of the current density, while at longer times, the slope is larger. For these high temperatures, the current does not tend to stabilize, even after 3 hours of applied voltage.

These differences in the current transient behaviour at different temperatures are certainly linked to different dominant processes. Current vs. time curves have been analysed in the literature, for HVDC terrestrial cable materials [17]. Polarization processes, linked to polar species, have been 
proposed as the dominant process at low temperatures, as conduction and/or injection processes do not contribute significantly to the current. At higher temperatures, polar contribution to current dominate at short times, but rapidly conduction becomes the dominant process. The same kind of hypotheses could hold in the case of PI, as PI is a polar material. Comparing polarization currents to depolarization currents (not presented here) strengthens the hypothesis that conduction processes are dominant for electric fields above $40 \mathrm{kV} / \mathrm{mm}$ at $20^{\circ} \mathrm{C}$. It is however difficult in the present case to decipher if conduction arises from ionic of electronic species, as it has been proposed in the literature that ions may be present and participate to conduction [13]. Many hypotheses have been proposed in the literature to interpret DC current behaviour in $\mathrm{PI}$, ranging from dipolar processes, to interface processes (injection), or to volume processes (Poole-Frenkel, ionic conduction....) [13-15]. However, these studies have been performed on different types of PI for different experimental protocols of voltage application, and most importantly various times under voltage application, ranging from $300 \mathrm{~s}$ to some hours. Our purpose here is only to highlight that for all temperatures, for relatively low to high electric fields, the current is thought to be dominated by conduction processes.

To strengthen this hypothesis, the current density has been plotted as a function of electric field for low temperatures $(20$, 50 and $90^{\circ} \mathrm{C}$ ) and is presented in Figure 5. Each current value is the mean of the currents over the last 2 minutes of the $3 \mathrm{~h}$ recording time at each voltage step. The feature current/electric field shows two regions separated by a threshold situated at 40 $\mathrm{kV} / \mathrm{mm}$. For electric fields lower than $40 \mathrm{kV} / \mathrm{mm}$, there is a slow increase of current, with a calculated slope in a log-log plot around 1. PI is thought to have an ohmic behaviour for fields below $40 \mathrm{kV} / \mathrm{mm}$ [5]. For electric fields higher than 40 $\mathrm{kV} / \mathrm{mm}$, the slope is over 3 (3.36). Considering the Space Charge Limited Current (SCLC) theory [8], the second slope is not linked either to a space charge limited region (slope normally equal to 2) or a trap filled limited region. However, depending on the physical processes at play (injection, transport of electronic, ionic species, trapping...), values of the slopes can differ from the theory. For all measured field values, there is no difference of conductivity value for temperatures in the range $20-90{ }^{\circ} \mathrm{C}$, i.e. conductivity is not evidently temperature dependent for temperatures below $90{ }^{\circ} \mathrm{C}$. The threshold in electric field appears at about $40 \mathrm{kV} / \mathrm{mm}$ in all cases.

To evaluate the dependence of conductivity on temperature, the current density has been measured as a function of temperature up to $200{ }^{\circ} \mathrm{C}$ for two electric fields (Figure 6), choosing-a low electric field $(20 \mathrm{kV} / \mathrm{mm})$, in the first region of Figure 5, and a higher electric field $(100 \mathrm{kV} / \mathrm{mm})$, in the second region of Figure 5 . At $20 \mathrm{kV} / \mathrm{mm}$, for temperatures below $120^{\circ} \mathrm{C}$, the current seems constant. Above this temperature value, the current tends to increase. At $100 \mathrm{kV} / \mathrm{mm}$, the threshold in temperature is observed at around $100^{\circ} \mathrm{C}$. In the literature, several reports are available on the behaviour of PI under thermo-electrical stress [5, 13-15], but the materials and experimental protocol differ from the ones used the present study. Motyl et al [5] reported on a threshold in current-field characteristic for all temperatures; this threshold value decreases when the temperature increases, to reach $40 \mathrm{kV} / \mathrm{mm}$

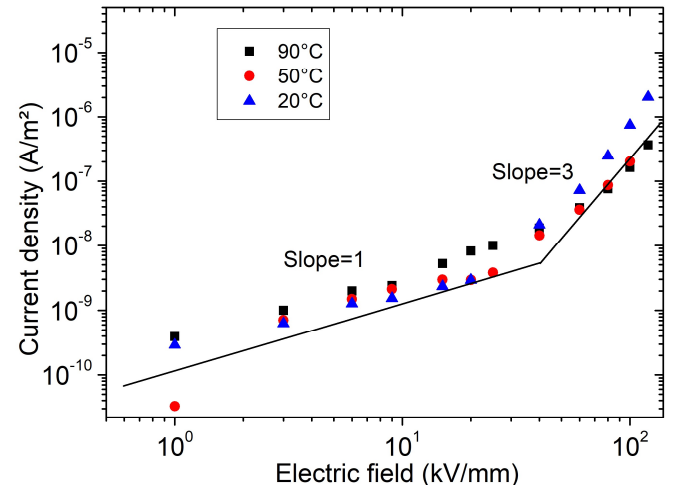

Figure 5. Current density versus electric field for different values of temperature $\left(20,50\right.$ and $\left.90^{\circ} \mathrm{C}\right)$ for $\mathrm{PI}$.

at $160^{\circ} \mathrm{C}$. A threshold in temperature is reported at $110^{\circ} \mathrm{C}$ for a field of $100 \mathrm{kV} / \mathrm{mm}$, moved to $160^{\circ} \mathrm{C}$ under $20 \mathrm{kV} / \mathrm{mm}$. The behaviour of this material is not far from Kapton-FN used in the present study, regarding the temperature threshold, but differs slightly when looking at the electric field threshold.

\subsection{CURRENT MEASUREMENTS ON PTFE SAMPLES}

The same kind of measurement has been performed for PTFE samples using the same two protocols.

Figure 7 shows current transients as a function of time for various electric fields applied on PTFE sample at a fixed temperature $\left(20^{\circ} \mathrm{C}\right)$. Current trend is decreasing and shows two different zones: one of sharp decrease and another in which current decreases more slowly and does not seem to stabilize at long times. This slope break depends on electric field as it appears earlier when the applied electric field is increased. At longer times, the current seems to stabilize only for the highest electric field after 3 hours of polarization. Nevertheless, the higher the value of the electric field, the more the current seems to be moving towards a stationary value.

Figure 8 presents the current density versus electric field obtained for different temperatures on PTFE samples. Current density increases according to the electric field. A threshold in field appears at $100 \mathrm{kV} / \mathrm{mm}$, i.e. at a higher value than the one observed for PI. The first slope (below $100 \mathrm{kV} / \mathrm{mm}$ ) is also 1 , i.e. PTFE has an ohmic behaviour up to relatively high applied

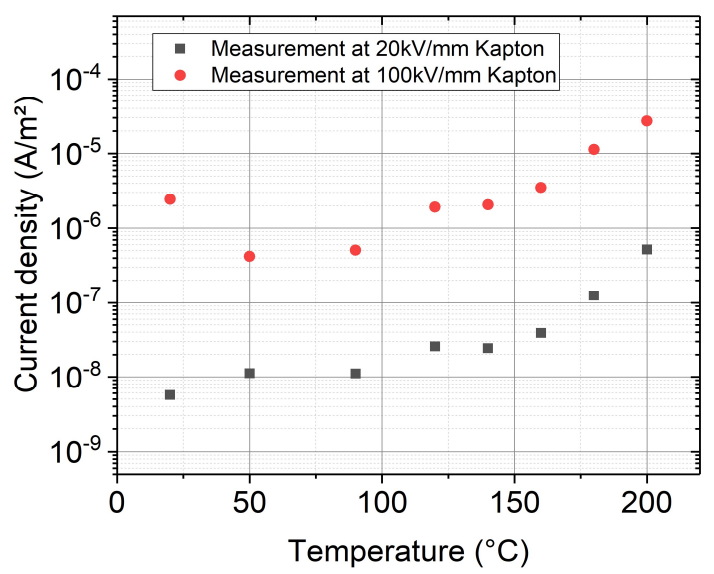

Figure 6. Current density versus temperature for two applied fields ( 20 $\mathrm{kV} / \mathrm{mm}$ and $100 \mathrm{kV} / \mathrm{mm}$ ) for PI 
electric fields, while the second slope is steeper, of the order of 5.8. For the tested temperatures, ranging from 20 to $90^{\circ} \mathrm{C}$, the current seems temperature independent. Current has also been measured for temperature values above $90^{\circ} \mathrm{C}$, for two applied electric fields only, to investigate the impact of temperature on electrical conductivity. Figure 9 shows current density as a function of temperature for each applied electric field (20 $\mathrm{kV} / \mathrm{mm}$ and $140 \mathrm{kV} / \mathrm{mm}$ in the case of PTFE).

At $20 \mathrm{kV} / \mathrm{mm}$, the current varies slightly with temperature. It is however difficult to discriminate if these variations are due to the measurement error, or if there is a real increase of the current for temperatures above $150^{\circ} \mathrm{C}$. For an applied field of $140 \mathrm{kV} / \mathrm{mm}$, it becomes clear that two regions appear, separated by a threshold at around $90^{\circ} \mathrm{C}$.

In the literature, the electrical properties of PTFE were particularly investigated with current measurements, although it is not clear if the material under study is the same PTFE as the one measured in this paper. As an example, for PTFE samples of $130 \mu \mathrm{m}$ thickness metallized with silver electrodes, Sussi et al [6] measured the current as a function of electric fields, for fields up to $25 \mathrm{kV} / \mathrm{mm}$ and temperatures ranging from 40 up to $200^{\circ} \mathrm{C}$. They observed three different regions in the JE behaviour, with an ohmic regime measured below an applied field value of $2 \mathrm{kV} / \mathrm{mm}$. These data are far from our experimental values.

PTFE samples of $50 \mu \mathrm{m}$ thickness, metallized with aluminium electrodes and placed in an oil bath, have also been characterized using current measurement at high electric fields (10 up to $400 \mathrm{kV} / \mathrm{mm}$ ) and low temperatures (up to $40^{\circ} \mathrm{C}$ ) [7]. The J-E characteristic obtained with these measurements shows four regions. At $20^{\circ} \mathrm{C}$, the electric field thresholds [7] are located at $20 \mathrm{kV} / \mathrm{mm}, 100 \mathrm{kV} / \mathrm{mm}$ and $200 \mathrm{kV} / \mathrm{mm}$. For electric field below $20 \mathrm{kV} / \mathrm{mm}$ the conduction current is ohmic. The second region is assigned to Poole-Frenkel effect. For an electric field between 100 and $200 \mathrm{kV} / \mathrm{mm}$, the current is attributed to Schottky effect and region 4 seems to be tunnelling current (Fowler-Nordheim effect) [7]. These different threshold values decrease with the increase in temperature. According to our results, there is only one threshold, appearing at $100 \mathrm{kV} / \mathrm{mm}$ at $20^{\circ} \mathrm{C}$ as an example. It may correspond to the second threshold reported in reference [7]. This different behaviour can be due to the fact that the nature of PTFE or the measurement conditions are not similar to ours. It is however difficult to find

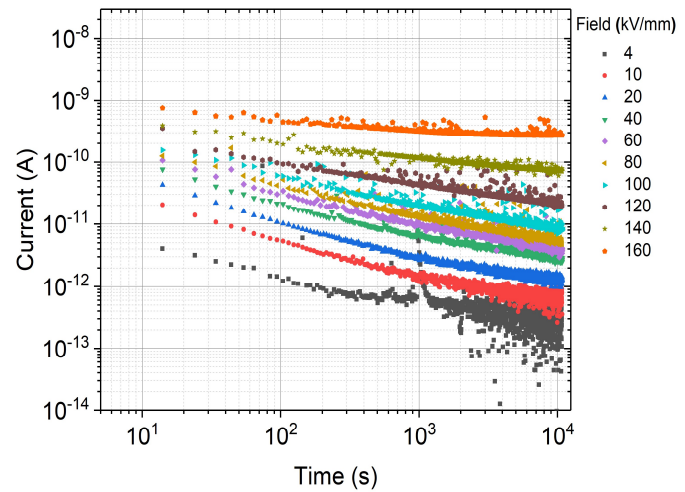

Figure 7. Charging current as function of time for different electric fields at $20^{\circ} \mathrm{C}$ on PTFE.

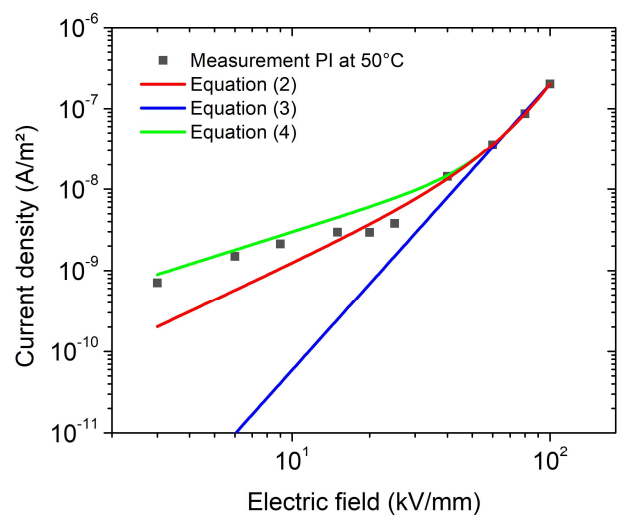

a) Current-density vs. field characteristic

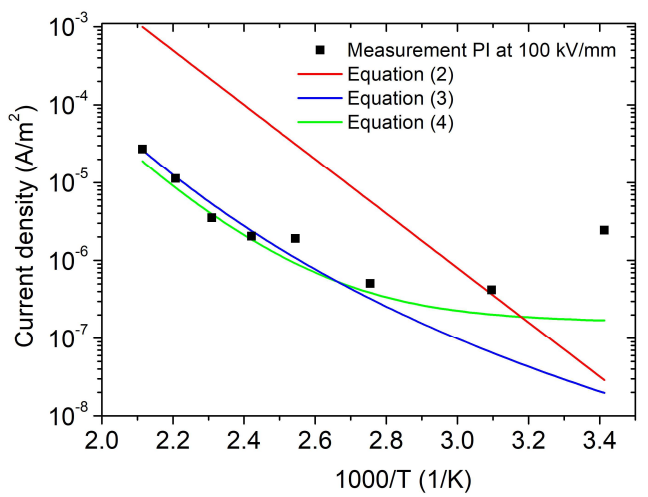

b) Current-density vs. temperature in Arrhenius plot

Figure 10. Fitted and experimental current density for PI according to models of Equations (2) to (4).

studies in the literature that report on the current at the same time at high fields and high temperatures, in the same experimental conditions (gold electrodes).

\section{ELECTRICAL CONDUCTIVITY LAW FOR MATERIALS}

A large number of equations can be found in the literature to describe the dependence of conductivity versus field and temperature of dielectric materials. Experimental data have clearly pointed out conductivity processes at high electric fields/ high temperatures, for both materials. As it is difficult to segregate injection-controlled processes from bulk-limited

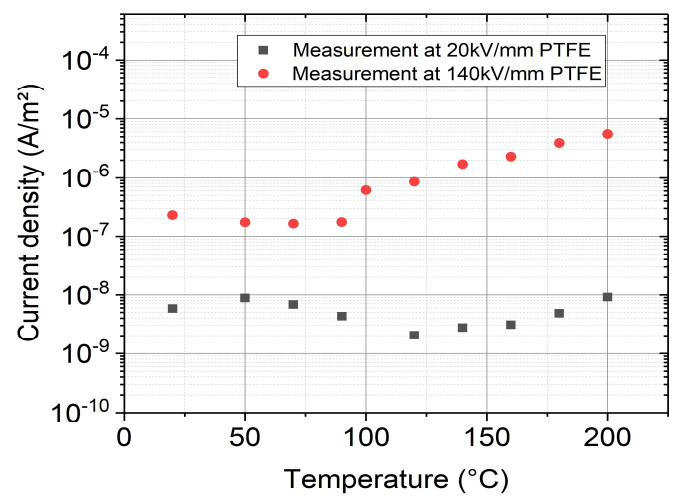

Figure 9. Current density versus temperature for different electric field (20 $\mathrm{kV} / \mathrm{mm}$ and $140 \mathrm{kV} / \mathrm{mm}$ ) on PTFE. 
conduction processes, injection processes such as FowlerNordheim or Schottky have been applied to our experimental data. Although these equations could fit the current-field characteristic for a specific temperature, the difference increases when the temperature increases. Hence, the current density behaviour cannot be approximated using charge injection equations. Conductivity laws have been applied to the experimental data, and among them one can cite [18, 19]:

$$
\begin{gathered}
\sigma(T, E)=C \exp \left(-\frac{E_{a}}{k_{b} T}\right) \sinh (D(T) E) E^{-1} \\
\sigma(T, E)=C \exp \left(-\frac{E_{a}}{k_{b} T}\right) \sinh (D(T) E) E^{\gamma} \\
\sigma(T, E)=\sigma_{\text {ref }}\left(\frac{E}{E_{\text {ref }}}\right)^{v} \exp \left[\beta\left(T-T_{\text {ref }}\right)\right]
\end{gathered}
$$

where $\mathrm{T}$ is the temperature, $\mathrm{k}_{\mathrm{B}}$ the Boltzmann's constant, $\mathrm{E}$ the electric field, $E_{a}$ an activation energy, $D(T)$ a temperaturedependent parameter, $\gamma$ a constant, $\mathrm{E}_{\text {ref }}$ the reference electric field, $\sigma_{\text {ref }}$ the reference electrical conductivity, $T_{\text {ref }}$ the reference temperature. Equation (1) is directly linked to a physical process (hopping mechanism [17]). Equation (2) is derived from Equation (1), while Equation (3) is with less obvious link with physical processes at play in the dielectric. Figure 10a) presents the above equations applied to current density vs. electric field for PI at a temperature of $50^{\circ} \mathrm{C}$. Parameters used for the different equations are presented in Table 1. Equation (3) clearly differs from experimental data at low fields, while equation (2) correctly fits the experiment. This is an expected result as regards the non-linear behaviour (log-log scale) of the experimental current density as a function of electric field. Figure 10b) presents the current density vs. reciprocal temperature for the different equations under study, for PI. On the contrary of Figure 10a), for Figure 10b) equation (3), with an exponential associated to the temperature, seems to better fit the experimental data for a specific electric field $(100 \mathrm{kV} / \mathrm{mm})$. None of these equations correctly fits our experimental data, for PI. The same is also true for PTFE (not shown here). Hence, another equation is proposed to fit electrical conductivity for PI and PTFE:

$$
\sigma(T, E)=A\left(1+\exp \left(B\left(T-T_{0}\right)\right)\right)\left(1+\left(\frac{E}{E_{0}}\right)^{\alpha}\right)
$$

The parameters of Equation (4), A, B, T, $\mathrm{E}_{0}$ and $\alpha$, are constants, and detailed in Table 1 for each material. Figure 10a presents the comparison of model/experimental current densities for PI as a function of electric field for a temperature of $50^{\circ} \mathrm{C}$. With Equation (4) and parameters from Table 1, simulation results are consistent compared to experiment over the range of electric fields under study. This holds for temperatures ranging from 20 up to $90^{\circ} \mathrm{C}$. It is also the case for current density as a function of temperature $(1000 / \mathrm{T})$ for an electric field $(100 \mathrm{kV} / \mathrm{mm})$, as equation (4) is the one that better reproduce the experimental data on Figure 10b). The same holds for a lower electric field.

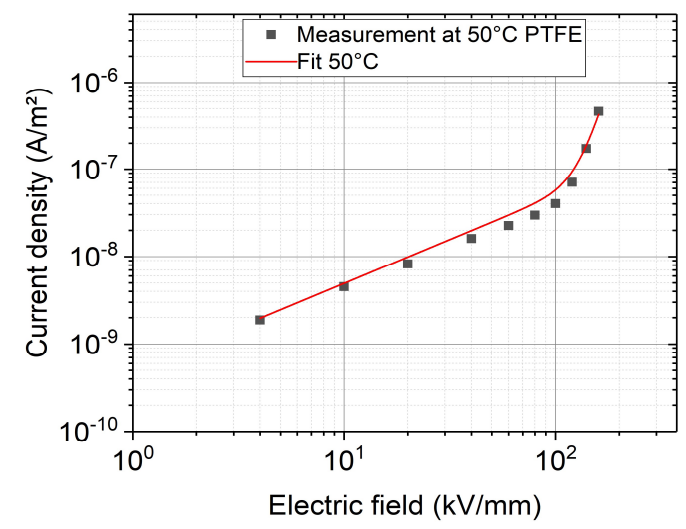

a) Current-field characteristic

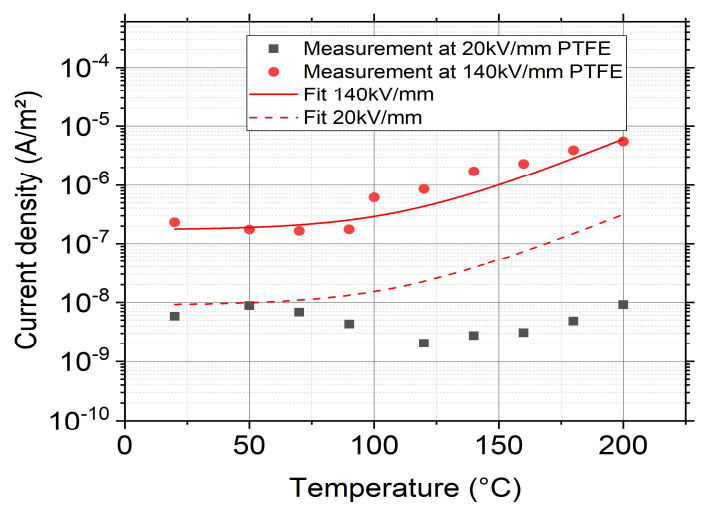

\begin{tabular}{|c|c|c|c|}
\hline Equations & Parameters & PI & PTFE \\
\hline \multirow{5}{*}{ Equation (4) } & $\mathrm{A}(\mathrm{S} / \mathrm{m})$ & $2.3 \times 10^{-16}$ & $4.5 \times 10^{-16}$ \\
\hline & To $\left({ }^{\circ} \mathrm{C}\right)$ & 80.5 & 106.7 \\
\hline & $\mathrm{B}\left({ }^{\circ} \mathrm{C}^{-1}\right)$ & 0.04 & 0.04 \\
\hline & Eo $(\mathrm{kV} / \mathrm{mm})$ & 60 & 130 \\
\hline & $\alpha$ & 3.49 & 6.97 \\
\hline \multirow{5}{*}{ Equation (3) } & $\sigma_{\text {ref }}(\mathrm{S} / \mathrm{m})$ & $8.92 \times 10^{-18}$ & $2.71 \times 10^{-18}$ \\
\hline & $\operatorname{Tref}\left({ }^{\circ} \mathrm{C}\right)$ & 22.3 & 22 \\
\hline & $\mathrm{E}_{\mathrm{ref}}(\mathrm{kV} / \mathrm{mm})$ & 18.2 & 79.2 \\
\hline & $\beta$ & 0.04 & 0.022 \\
\hline & $v$ & 2.5 & 5.7 \\
\hline \multirow{4}{*}{ Equation (2) } & $\mathrm{C}$ & 0.14 & 22.5 \\
\hline & $\mathrm{D}(\mathrm{m} / \mathrm{V})$ & $3.68 \times 10^{-8}$ & $3.3 \times 10^{-8}$ \\
\hline & $\mathrm{Ea}(\mathrm{eV})$ & 0.71 & 0.41 \\
\hline & $\gamma$ & -0.51 & -0.58 \\
\hline
\end{tabular}

b) Current-temperature characteristic

Figure 11. Fitted and experimental current density for PTFE.

Table 1. Parameters for electrical conductivity laws.

For PTFE, experimental and fitted data are compared in Figure 11a as a function of applied fields for a low temperature, and in Figure $11 \mathrm{~b}$ as a function of temperature for low and high electric fields. At low temperature (Figure 11a), the variation of conductivity with electric field is well reproduced. The agreement between model and experimental data at higher temperature for fixed electric fields (Figure 11b) is fairly consistent for a high electric field, but not for $20 \mathrm{kV} / \mathrm{mm}$, where 
the conductivity is almost constant. For this electric field, the fit is consistent for low temperatures, but diverges from experimental data when the temperature increases to reach a conductivity that is a decade higher than the experimental one.

One must question about the validity of the model as well as the experiments for the difficulty in identifying a model for PTFE. Regarding experiments, obtained currents are larger than the detection limits of the set-ups (cf. case of PI), therefore it seems not the limiting factor. The second possibility is material modification during experiments due to thermal conditioning. Humidity variation, morphological evolution are possible reasons. This seems unlikely here as PTFE is hydrophobic material, and sintering was applied before measurements, meaning that stabilization of the structure has occurred. The last potential reason is, as discussed previously, the necessary limitation in time of the measurements and therefore the possibility that different processes are dominant at different time scales, depending on the temperature. This makes the link with questioning about the model.

Equation (4) that is used to fit experimental current density is composed of two parts. The first part describes temperaturedependence of electrical conductivity. The function that has been used does not include a thermal activation energy with Arrhenius law but accounts for a temperature threshold and temperature dependence of conductivity beyond the threshold. The function for the electric field dependence is defined by a power law in field above a given threshold, consistently with previous expressions and following the classical way of interpreting current-field characteristics. The chosen expression assumes that there is no simple /dominant process governing the macroscopic conduction, amongst those found in the literature with different formulas reflecting Schottky emission, Poole-Frenkel or other mechanisms. It globally fits all experimental data.

As such, Equation (4) decouples temperature and field effects. It means that a temperature dependent field threshold for nonlinear conduction for example, as was reported elsewhere for other materials [19] cannot be reproduced unless parameters of the equation become temperature dependent. At present data on PI or PTFE are not enough documented for introducing such effect.

Looking at the parameters of Table $1, \mathrm{~A}$ is a constant that describes the initial electrical conductivity value when field is very low and temperature is low. $\mathrm{T}_{0}$ defines the threshold at which conductivity begins to increase with temperature and $\mathrm{B}$ the rate of increase of conductivity beyond this threshold. At the level of electric field dependence of conductivity, $\mathrm{E}_{0}$ defines the field threshold for non-linear conductivity and $\alpha$ reflects the rate of change of conductivity beyond this field. At the temperature or field threshold, the conductivity becomes twice as the one at low field/low temperature. This provides an easy way to compare the characteristics of different materials. The dielectric permittivity is lower (i.e. more favourable for electrical insulation purpose) in PTFE $\left(\varepsilon_{\mathrm{r}}=2.1\right.$ [20]) compared to PI $\left(\varepsilon_{\mathrm{r}}=3.4[11]\right)$. The temperature threshold is lower $\left(80^{\circ} \mathrm{C}\right)$ for PI compared to PTFE $\left(106.7^{\circ} \mathrm{C}\right)$, which reflects the difficulty in establishing a clear temperature dependence of conductivity for PTFE. Regarding the field threshold, it is substantially higher for PTFE $(130 \mathrm{kV} / \mathrm{mm})$ compared to PI
$(60 \mathrm{kV} / \mathrm{mm})$. Therefore, although the low field / low temperature conductivity is about twice in PTFE compared to PI, the former seems to have better DC insulation performances.

A further criterion regarding performances is the breakdown strength. It is well known that values are extremely dependent on the insulation thickness, on sample area and on test methodology [21]. DC breakdown strength beyond $600 \mathrm{kV} / \mathrm{mm}$ has been reported both for PI and PTFE for films thicknesses in the range 5 to $25 \mu \mathrm{m}[22,23]$, indicating high performances for both materials. However, there is no available parallel study on breakdown strength and its temperature dependence realized in similar conditions. Such analysis would be interesting in parallel to conductivity measurements since thermal breakdown is one of the possible breakdown process [24] aside spacecharge induced breakdown [25].

\section{CONCLUSIONs}

Current measurements have been performed for PI and PTFE materials, both used as insulation in HVDC aeronautic cables, in order to characterize their behaviour as regards electric field and temperature. The current/field characteristics show a threshold in field at around $40 \mathrm{kV} / \mathrm{mm}$ for PI and at $100 \mathrm{kV} / \mathrm{mm}$ for PTFE, for relatively low temperatures up to $90^{\circ} \mathrm{C}$. According to the results on PI, the threshold in temperature is at around $160^{\circ} \mathrm{C}$ and $110^{\circ} \mathrm{C}$ respectively for $20 \mathrm{kV} / \mathrm{mm}$ and $100 \mathrm{kV} / \mathrm{mm}$. From these experimental data, a conductivity law has been found to better fit the experimental characteristics of both materials. Although PTFE shows no variation of conductivity at low fields over the range of tested temperatures, there is a threshold in temperature for higher fields. These experimental data need to be strengthened for a larger number of electric field values for the tested range of temperatures. The conductivity law will also serve as an input in a macroscopic model to simulate the electric field distribution and interfacial charge accumulation in real cable geometries to understand the electric field behaviour in aeronautic cables under thermoelectric stress.

\section{REFERENCES}

[1] V. Madonna, P. Giangrande, and M. Galea, "Electrical power generation in aircraft: Review, challenges, and opportunities," IEEE Trans. Transp. Electrific., vol. 4, pp. 646-659, 2018.

[2] B. Sarlioglu and C. T. Morris, "More electric aircraft: Review, challenges, and opportunities for commercial transport aircraft,", IEEE Trans. Transp. Electrific., vol. 1, pp. 54-64, 2015.

[3] W. Xin, R. Mahmoudi, and C. E. Lents, "A Study of partial discharge in high voltage DC distribution systems for hybrid electric aircraft," 2018 AIAA/IEEE Electr. Aircr. Technol. Symp., 2018, pp. 1-9.

[4] G. C. Montanari, "The electrical degradation threshold ofpolyethylene investigated by space charge and conductioncurrent measurements," IEEE Trans. Dielectr. Electr. Insul.,vol. 7, no. 3, pp. 309-315, 2000.

[5] E. Motyl and R. Kacprzyk, "On the electrical conduction in polyimide," IEEE Int. Sympos. Electrets (ISE), 1996, pp. 608-613.

[6] M. A. Sussi and G. R. G. Raju, "Electrical conduction currents in polytetrafluoroethylene," Annu. Rep. Conf. Electr. Insul. Dielectr. Phenomena (CEIDP), 1990, pp. 313-318.

[7] J. U. Lee, C. H. Kim, S. Lee, J. B. Wang, D. Kim, and S. S. Lee, "Nonlinear conduction properties of polytetrafluoroethylene film," IEEE Internat. Symp. Electr. Insul. (ISEI), 1992, pp. 149-152.

[8] L. A. Dissado and J. C. Fothergill, Electrical Degradation and Breakdown in Polymers, Peter Peregrinus, 1992. 
[9] P. R. Hondred, N. Bowler, and M. R. Kessler, "Electrothermal lifetime prediction of polyimide wire insulation with application to aircraft," $\mathrm{J}$. Appl. Polym. Sci., vol. 130, pp. 1639-1644, 2013.

[10] X. Huang, J. Martinez-Vega, and D. Malec, " Morphological evolution of polytetrafluoroethylene in extreme temperature conditions for aerospace applications," J. Appl. Polymer Sci., vol. 131, p. 39841, 2013.

[11] L.W. McKeen, "Polyimides," in Plastics Design Library: Film Properties of Plastics and Elastomers (fourth edition), William Andrew Publishing, Ed. L.W. McKeen, 2017, pp.147-185.

[12] H. Ghorbani et al, "Long-term conductivity decrease of polyethylene and polypropylene insulation materials," IEEE Trans. Dielectr. Electr. Insul, vol. 24, no. 3, pp. 1485-1493, 2017.

[13] B. L. Sharma and K. C. Pillai, "Electrical conduction in Kapton polyimide film at high electric fields," Polymer, vol. 23, pp. 17-20, 1982.

[14] Y. Segui, H. Benalla, and J. Farenc, "Electrical conductivity of poly (amide-imide) films," Internat. Conf. Conduction and Breakdown in Solid Dielectrics, 1983, pp. 124-128.

[15] G. M. Sessler, B. Hahn, and D. Y. Yoon, "Electrical-conduction in polyimide films", J. Appl. Phys., vol. 60, pp. 318-326, 1986.

[16] Y. P. Khanna, "The melting temperature of polytetrafluoroethylene," J. Mater. Sci. Lett., vol. 7, no. 8, pp. 817-818, 1988.

[17] J. Y. Steven, T. T. N. Vu, G. Teyssedre, and N. I. Sinisuka, "Conductivity measurements and space charge inference in polymeric-insulated HVDC model cables," IEEE Int. Conf. High Voltage Eng. Appl. (ICHVE), 2014, pp. $1-4$.
[18] P.H.F. Morshuis et al, "Calculation and measurement of space charge in MV-size extruded cables systems under load conditions," IEEE Int.. Conf. Solid Dielectr. (ICSD), 2007, vol. 2, pp. 502-505.

[19] T. T. N. Vu, G. Teyssedre, B. Vissouvanadin, S. Le Roy, and C. Laurent, "Correlating conductivity and space charge measurements in multidielectrics under various electrical and thermal stresses," IEEE Trans. Dielectr. Electr. Insul, vol. 22, no. 1, pp. 117-127, 2015.

[20] L. Li. "Dielectric properties of aged polymers and nanocomposites," $\mathrm{PhD}$ dissertation, Dept. Mater. Sci. Eng., Iowa State Univ., USA, 2011.

[21] S. Diaham et al, "Dielectric breakdown of polyimide films: Area thickness and temperature dependence," IEEE Trans. Dielectr. Electr. Insul., vol. 17, no. 1, pp. 18-27, 2010.

[22] D. Min et al, "Thickness-dependent DC electrical breakdown of polyimide modulated by charge transport and molecular displacement," Polymers, vol. 10, pp. 1012-1029, 2018.

[23] C. M. Kerwien, D. L. Malandro, and J. R. Broomall, "Large area DC dielectric breakdown voltage measurement of BOPP and PTFE thin films," Annu. Rep. Conf. Electr. Insul. Dielectr. Phenomena (CEIDP), 2016, pp. 486-489.

[24] M. Nagao, G. Sawa, M. Fukui, and M. Ieda, "Dielectric breakdown of polyimide film in high temperature region," Jap. J. Appl. Phys., vol. 15, pp. 1813-1814, 1976.

[25] S. Li, Y. Zhu, D. Min, and G. Chen, "Space charge modulated electrical breakdown," Sci. Rep., vol. 6, pp. 32588, 2016. 